\title{
Estrés académico y resiliencia en madres universitarias colombianas con y sin apoyo institucional en el cuidado de sus hijos*
}

\author{
Academic stress and resilience in university Colombian mothers with \\ and without institutional support in the care of their children
}

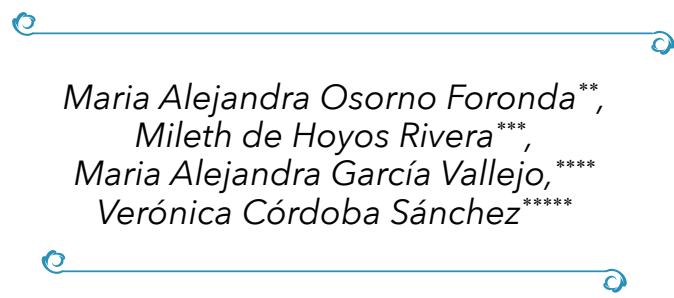

* El presente artículo es producto de la tesis de grado para optar al título de Psicología de la Institución Universitaria de Envigado, el cual se tituló: Niveles de estrés académico y resiliencia en madres universitarias con y sin apoyo institucional en el cuidado de sus hijos del Instituto Tecnológico Metropolitano y la Institución Universitaria de Envigado.

* Institución Universitaria de Envigado, Facultad de Ciencias Sociales, Envigado - Colombia. https:/ / orcid. org/0000-0003-0975-2447

** Institución Universitaria de Envigado, Facultad de Ciencias Sociales, Envigado - Colombia. https://orcid. org/0000-0001-8027-9045

*** Institución Universitaria de Envigado, Facultad de Ciencias Sociales, Envigado - Colombia. https:/ / orcid. org/0000-0003-0287-2155

Institución Universitaria de Envigado, Facultad de Ciencias Sociales, Envigado - Colombia. https:/ / orcid. org/0000-0001-6037-1629

\section{Resumen}

Son cada vez más las mujeres que asumen simultáneamente los roles de madre y estudiante en el ámbito universitario; lo cual puede hacerlas vulnerables a factores que obstaculizan su adecuado proceso académico. A través de un estudio cuantitativo, con un diseño de casos y controles, se analizó la asociación entre el estrés académico y la resiliencia en una muestra de 52 madres de dos instituciones universitarias colombianas: el Instituto Tecnológico Metropolitano y la Institución Universitaria de Envigado, de las cuales, la primera cuenta con un aula pedagógica de apoyo en el cuidado de los hijos de las estudiantes y se constituyó en el grupo de casos y la segunda no cuenta con este beneficio, conformando el grupo de control. Para la recolección de datos se aplicó un cuestionario sociodemográfico, la Escala de 
Resiliencia SV-RES y el Inventario de Estrés Académico SISCO. Se halló que el grupo de control tuvo niveles superiores de estrés psicológico y presentó una asociación negativa entre el estrés psicológico, comportamental y físico y el nivel total de resiliencia.

Palabras clave: estrés académico, estudiantes universitarios, maternidad, resiliencia, mujeres.

\section{Abstract}

Women who simultaneously assume the roles of mother and student in the university environment are more and more frequent, which can make them vulnerable to factors that hinder their proper academic process. Through a quantitative study with a case-control design, we analyzed the association between academic stress and resilience in a sample of 52 mothers from two Colombian universities: The Instituto Tecnológico Metropolitano, and the Institución Universitaria de Envigado, the first has a support pedagogical classroom in the care of the students' children and was constituted in the group of cases and the second does not have this benefit, forming the control group. For data collection, we used a sociodemographic questionnaire, the SV-RES Resilience Scale and the SISCO Academic Stress Inventory. We found that the control group had higher levels of psychological stress and in this group, there was a negative association between psychological, behavioral, and physical stress and the total level of resilience.

Keywords: Academic stress, university students, motherhood, resilience, women.

\section{Introducción}

En la actualidad muchas mujeres universitarias conjugan su rol de estudiantes con el de madres, situación que puede llegar a afectar su experiencia académica, no solamente en cuanto a su rendimiento sino también en los aspectos emocionales y sociales, al poner en riesgo su salud física y mental, así como el adecuado desarrollo de sus hijos (Estupiñán y Vela, 2012).

Para las madres universitarias ambos roles pueden llegar a ser igualmente importantes; sin embargo, en su caso se ve más afectado el de estudiante, pues tienden a llevar una carga académica inferior a la que formalmente debería ser, lo cual tendría como principal consecuencia que se tarden más tiempo en terminar la carrera que otras estudiantes (Rodríguez et al., 2019). Esto puede estar relacionado con que, en Colombia, como en otros países de la región, el cuidado de los menores recae especialmente en las mujeres y es una actividad que ocupa gran cantidad de tiempo. 
El Departamento Administrativo Nacional de Estadística (DANE) y onu Mujeres (2020) reportan que en el país 29.8 millones de personas realizan actividades de cuidados de algún tipo al interior de sus hogares, de los cuales las mujeres realizan $76,2 \%$ de ese trabajo. Se trata de una labor que generalmente consiste en el cuidado niños, niñas y adolescentes entre los 0 y los 14 años, es decir el $90,5 \%$ de todas las personas que reciben cuidado en Colombia. Asimismo, las entidades mencionadas resaltan en su informe que quienes más sufren del fenómeno denominado "pobreza de tiempo" son las mujeres, puesto que el tiempo que destinan a proveer cuidados interfiere con el desarrollo de actividades en otras esferas de la vida, entre las cuales se encuentra el estudio.

Esta situación puede obstaculizar que las mujeres alcancen su máximo potencial y cuenten con la formación adecuada para acceder a un trabajo remunerado y proveer bienestar a sus hijos, pues se ha identificado que la educación es el mecanismo central de cambio de las situaciones de marginalidad, dado que le confiere a las mujeres no solo la calificación para desarrollar una actividad productiva sino también para tener conciencia de sus derechos, así como autoestima y control sobre las condiciones en las que transcurre su propia vida (CEPAL, 1998).

Para la mayoría de las personas, la educación universitaria supone un proceso lleno de retos, aprendizajes y, sobre todo, exigencias de adaptación a un ambiente académicamente competitivo, en el cual se convive con situaciones como el cumplimiento de horarios de clase y actividades evaluativas. Esta situación es condicionada por diversos factores psicosociales que, de acuerdo con investigaciones locales recientes, pueden constituirse en factores generadores de estrés (Restrepo et al., 2020).

El concepto clásico de estrés de Lazarus y Folkman (1986) alude a la manifestación de dos procesos psicológicos que ocurren frente a un suceso: en primer lugar, su evaluación cognitiva y en segundo, la respuesta de afrontamiento. A su vez Sapolsky (2008) refiere que la respuesta de estrés es la intención del organismo por recuperar la alostasis o equilibrio interno del cuerpo ante un estímulo estresante.

Al respecto Oken et al. (2014), en línea con Sapolsky (2008), plantean que el estrés no es perjudicial en sí mismo, sino que el problema está en la incapacidad de poder recuperar el equilibrio luego de perderlo. Es decir que el problema es la disminución de la eficacia para retornar o permanecer en la línea de base de funcionamiento positivo al experimentar situaciones estresantes. Esto ocurre cuando se tiene baja resiliencia, pues esta determina qué tan eficaz y rápidamente el sistema regresa a la línea de base después de una perturbación. 
La resiliencia se refiere a la capacidad que tienen las personas para mantener un funcionamiento psicológico y físico aun cuando son sometidas a situaciones traumáticas o altamente estresantes (Russo et al., 2012). Este concepto trasciende la ausencia de psicopatología pues se le considera un proceso activo y de adaptación, del cual hacen parte rasgos como el uso de estrategias de afrontamiento activo, la capacidad de enfrentarse a los temores, la búsqueda de apoyo social, el optimismo, la aceptación, la valoración de los eventos negativos como poco amenazantes e incluso otros aún poco explorados como el sentido de propósito en la vida, la espiritualidad, una brújula moral y la capacidad de encontrar sentido a los eventos traumáticos (Feder et al., 2009).

El ámbito educativo no es ajeno al fenómeno del estrés, pues allí también puede ocurrir que los estudiantes, al valorar que sus dificultades sobrepasan sus recursos, sufran alteraciones del funcionamiento general y aparezcan síntomas cognitivos, emocionales y físicos. Estos pueden manifestarse en la disminución del rendimiento académico, la concentración, la motivación, en dificultades en la interacción con compañeros y profesores, ausentismo escolar, quejas somáticas, conductas ansiosas, labilidad emocional y alteraciones en el patrón del sueño. Estos síntomas configuran un fenómeno que recibe el nombre de estrés escolar (Barraza y Silerio, 2007; Maturana y Vargas, 2015; Martínez y Díaz, 2007) y que se ha evidenciado no solo en la educación básica, sino también en el nivel de formación universitario (Berrío y Mazo, 2011; González-Olaya et al., 2014).

El estrés en el contexto académico ha sido abordado progresivamente por la comunidad científica en los últimos años. En los principales hallazgos de diversas investigaciones, se destaca que las situaciones que más generan estrés en los estudiantes son la sobrecarga de actividades evaluativas y el tiempo limitado para hacerlas (Berrío y Mazo, 2011; Restrepo et al., 2020). Adicionalmente otro hallazgo significativo es que las mujeres suelen presentar mayores niveles de estrés que los hombres que están en similares circunstancias (González-Olaya et al., 2014). No obstante, en las madres universitarias este fenómeno se encuentra poco estudiado, pese a que se conoce que el estado de salud mental de la madre puede impactar en el desarrollo físico y psicológico de su hijo (Álamo et al., 2017; Steen y Francisco, 2019).

En los escasos antecedentes se subraya que en esta población se presenta deterioro en la salud física, estrés y depresión y que su calidad de vida está ligada a sus relaciones familiares y al apoyo social que perciben en el contexto universitario (Estupiñán y Vela, 2012). De igual forma, se ha encontrado que las mujeres que tienen esta condición presentan dificultad para combinar los roles de madre y estudiante debido a la falta de tiempo. En su relato se 
encuentra la obligación de tener horarios extensos para cubrir jornadas laborales, escolares y domésticas, lo que les trae como consecuencia bajo rendimiento escolar, dejar el cuidado de los hijos a terceros, aceptar trabajos que se ajustan a sus horarios o incluso suspender sus estudios (Miller y Arvizu, 2016; Domínguez et al., 2015).

No obstante, algunos estudios reportan que estas mujeres reconocieron que a partir de la maternidad se sentían capaces de afrontar grandes retos, de ser maduras y responsables y muy pocas consideraban que les hubiera gustado ser madres en otro momento de la vida (Urbano et al., 2012; Domínguez et al., 2015; Rodríguez et al., 2019). El hecho de enfocarse en las expectativas de vida y poder darles un buen futuro a sus hijos se convirtió en un motivador para conseguir las metas y perseverar en su consecución, siendo fundamental el papel de la resiliencia y el del apoyo familiar (Urbano et al., 2012). Se ha encontrado también que situaciones adversas como el embarazo y la maternidad en la adolescencia pueden movilizar mecanismos de resiliencia vinculados al rendimiento académico (Villalta, 2010).

Sin embargo, es importante reconocer que la institución a la cual se encuentran vinculadas tiene un papel fundamental para la culminación de sus estudios superiores y, en aras de fomentar la permanencia de las estudiantes, es necesario que se reconozcan sus características y posibilidades. De esta manera el objetivo del presente estudio fue determinar la asociación entre los niveles de estrés académico y la resiliencia en madres universitarias con y sin apoyo institucional en el cuidado de sus hijos.

\section{Metodología}

\section{Diseño}

Se desarrolló una investigación con enfoque cuantitativo, de nivel correlacional, con un diseño de casos y controles, en el que se analizó la asociación de dos grupos. Uno de los grupos estaba expuesto a una intervención institucional específica en el cuidado de los hijos (programa Aula Pedagógica Infantil), mientras que el otro grupo no contaba con dicho apoyo.

El programa Aula Pedagógica Infantil es un espacio creado para brindar atención, cuidado y acompañamiento pedagógico a los niños y niñas entre los 6 meses y los 3 años, hijos de estudiantes, empleados y contratistas del Instituto Tecnológico Metropolitano (ITM). Este programa tiene como objetivo favorecer el ingreso, la permanencia y la culminación del proceso formativo 
de los estudiantes, a través de un servicio de atención, cuidado y acompañamiento pedagógico para sus hijos menores de 3 años. Así mismo, busca aportar al cumplimiento del principio de corresponsabilidad establecido en el Código de Infancia y Adolescencia (Ley 1098 de 2006), que establece "la obligación y la responsabilidad de tomar parte activa en el logro de la vigencia efectiva de los derechos y garantías de los niños, las niñas y los adolescentes", favoreciendo la formación de adultos responsables de la educación de sus hijos y de su rol educativo (ITM, s.f.).

\section{Muestra}

La muestra estuvo conformada por 52 mujeres adultas que tenían al menos un hijo y que fueran estudiantes universitarias, las cuales fueron divididas en dos grupos, de la siguiente manera:

Grupo de casos: 26 mujeres del Iтм beneficiarias del programa Aula Pedagógica Infantil.

Grupo de control: 26 mujeres pertenecientes a la Institución Universitaria de Envigado (IUE), que tenían la misma edad y el mismo número de hijos que el grupo de casos y que no contaban con ayuda institucional para el cuidado de sus hijos.

\section{Instrumentos}

Datos sociodemográficos: se diseñó un cuestionario específico para identificar características sociales, económicas y familiares como la edad, número y edad de los hijos, estado civil, situación laboral, estrato socioeconómico, apoyo familiar, entre otros.

Escala de Resiliencia SV-RES (Saavedra y Villalta, 2008), la cual consta de 60 ítems, con respuesta tipo Likert. Este cuestionario fue diseñado para medir resiliencia en jóvenes y adultos, a nivel general y en doce factores específicos: identidad, autonomía, satisfacción, pragmatismo, vínculos, redes, modelos, metas, afectividad, autoeficacia, aprendizaje, generatividad. Los autores han reportado una validez externa con el cuestionario CD-RISC, con un coeficiente de correlación Pearson, obteniendo un $\mathrm{r}=, 75 \mathrm{y}$ una confiabilidad de Alfa de Cronbach $=0,96$ para la prueba de dos mitades (Saavedra y Villalta, 2008).

Inventario SISCO del estrés académico (Barraza, 2007), el cual busca reconocer las características del estrés que suelen acompañar a los estudiantes. Está compuesto por 41 ítems con respuesta tipo Likert, agrupados en tres dimensiones: situaciones estresantes, reacciones y estrategias (en el presente 
estudio se emplearon solamente las escalas de situaciones y reacciones). Barraza reportó un Alfa de Cronbach de 0,838 para la confiabilidad global y un y $\mathrm{r}=0,766$ para la confiabilidad por mitades. En Colombia fue validado por Malo, Cáceres y Peña (2010) en adultos jóvenes estudiantes universitarios quienes mostraron un alfa adecuado para la confiabilidad y valores apropiados para la validez (análisis factorial y grupos contrastados).

\section{Procedimiento}

La investigación se realizó cumpliendo con lo establecido en la Ley 1090 de 2006 que reglamenta el ejercicio de la profesión de la psicología y establece el correspondiente código deontológico y bioético, así como con la Resolución 8430 de 1993 del Ministerio de Salud por la cual se establecen las normas científicas, técnicas y administrativas para la investigación en salud. El proyecto se presentó a ambas instituciones, quienes dieron su aprobación para la ejecución y cada participante suministró su consentimiento informado por escrito.

Luego de la autorización institucional, se presentó el proyecto a todas las madres estudiantes beneficiarias del Aula Pedagógica Infantil y se les invitó a participar en el proyecto, tras lo cual se les entregaron los cuestionarios para su diligenciamiento.

Una vez se conformó la muestra del grupo de casos, se procedió a reclutar a las participantes de la IUE, las cuales serían el grupo de control, emparejadas con el grupo de casos por edad y número de hijos. El reclutamiento se hizo a través de un correo electrónico masivo y avisos en diferentes lugares de encuentro de la institución y se concertó con ellas un encuentro para la recolección de los datos.

En ambos grupos el instrumento fue autoadministrado y la recolección de datos se llevó a cabo entre agosto y septiembre de 2019.

\section{Análisis de datos}

Se realizó un análisis de datos de tipo casos y control. Los casos estaban expuestos a una intervención institucional específica en el cuidado de los hijos, a través del programa Aula Pedagógica Infantil, mientras que el otro grupo no contaba con dicho apoyo. De esta manera, la manipulación de la variable independiente alcanzó solo dos niveles: presencia y ausencia.

Se realizó un análisis de frecuencia y correlación con programas computacionales como el SPSS (Paquete Estadístico para las Ciencias Sociales) versión 25, para visualizar y analizar descriptivamente los datos, se utilizó la prueba 
Chi cuadrado $\left(\mathrm{X}^{2}\right)$ con el objetivo de hallar la significación asintótica y la prueba de Pearson para determinar el coeficiente de significación y determinar la correlación entre las variables de la presente investigación. Además, se incluyó también la prueba de Mann Whitman que se utilizó para correlacionar las diferentes dimensiones del estrés: estrés psicológico, comportamental y físico en ambos grupos.

\section{Resultados}

A continuación, se describen los hallazgos obtenidos de acuerdo con la pertenencia al grupo de casos que cuentan con apoyo institucional para el cuidado de los hijos o al grupo de control que no cuenta con este apoyo.

En la tabla 1 se muestra la frecuencia y los porcentajes de las características sociodemográficas y su coeficiente de significancia estadística entre el grupo de casos y el grupo de control. En estos resultados se destaca que, en cuanto a la vinculación laboral, en el grupo de casos, un 42,3\% de las madres trabajan frente a un 53,8\% del grupo de control; con respecto al estrato de la vivienda, se encontró que la mayoría de las participantes (50\%) residía en viviendas de estrato dos y en el grupo de control la mayoría $(46,2 \%)$ lo hacía en viviendas de estrato tres; en ambos grupos el 80,5\% de las mujeres tenía pareja.

En relación con las personas o familiares que les ofrecen apoyo en el cuidado de sus hijos, en el grupo de casos se encontró que 46,2\% de la muestra respondió que nadie la apoya frente a un 7,7\% en el grupo de control. En cuanto a quién es la persona o personas que apoyan, en el grupo de casos el 19,2\% respondió que el apoyo lo recibe por parte de su pareja y en el grupo de control el 50,0\% tuvo esta misma respuesta; en ambos grupos el 23,1\% de las participantes respondió que recibe apoyo de sus padres; de igual forma en el grupo de casos el 11,5\% respondió que este apoyo lo recibe de otros y en el grupo de control el 19,2\%; esta variable arrojó una significación estadística de 0,011.

Acerca de la consideración de abandono o aplazamiento de su formación académica por causa de la maternidad, en el grupo de casos el 76,9\% de las madres ha considerado abandonar o aplazar sus estudios y en el grupo de control lo ha considerado el $57,7 \%$ de las madres; con respecto al porcentaje de deserción o abandono efectivo de los estudios en el grupo de casos fue de un $69,2 \%$ y en el del grupo de control fue del 50\%; con referencia al apoyo familiar en el cuidado de los hijos, en el grupo de casos un 65,4\% respondió que sí lo tenía y en el grupo control un $88,5 \%$; esta variable tuvo un coeficiente de significación del 0,048, lo que muestra una diferencia estadísticamente significativa entre los dos grupos. 
Tabla 1. Datos sociodemográficos de las madres universitarias

\begin{tabular}{|c|c|c|c|c|c|c|c|}
\hline \multirow[t]{2}{*}{ Variable } & \multirow[t]{2}{*}{ Respuesta } & \multicolumn{2}{|c|}{$\begin{array}{l}\text { Grupo } \\
\text { Casos }\end{array}$} & \multicolumn{2}{|c|}{$\begin{array}{l}\text { Grupo } \\
\text { Control }\end{array}$} & \multirow[t]{2}{*}{ Chi $\chi^{2}$} & \multirow{2}{*}{$\begin{array}{l}\text { Significación } \\
\text { asintótica }\end{array}$} \\
\hline & & $\mathbf{n}$ & $\%$ & $\mathbf{n}$ & $\%$ & & \\
\hline \multirow{2}{*}{ Vinculación laboral } & Sí & 11 & 42,3 & 14 & 53,8 & \multirow{2}{*}{0,693} & \multirow{2}{*}{0,405} \\
\hline & No & 15 & 57,7 & 12 & 46,2 & & \\
\hline \multirow{5}{*}{ Estrato de la vivienda } & 1 & 2 & 7,7 & 1 & 3,8 & \multirow{5}{*}{6,341} & \multirow{5}{*}{0,175} \\
\hline & 2 & 13 & 50,0 & 6 & 23,1 & & \\
\hline & 3 & 9 & 34,6 & 12 & 46,2 & & \\
\hline & 4 & 2 & 7,7 & 6 & 23,1 & & \\
\hline & 5 & 0 & 0,0 & 1 & 3,8 & & \\
\hline \multirow{4}{*}{ Con quién convive } & Pareja e hijos & 16 & 61,5 & 13 & 50,0 & \multirow{4}{*}{2,669} & \multirow{4}{*}{0,445} \\
\hline & Familia de origen & 5 & 19,2 & 8 & 30,8 & & \\
\hline & Hijos solamente & 4 & 15,4 & 2 & 7,7 & & \\
\hline & Hijos y otros & 1 & 3,8 & 3 & 11,5 & & \\
\hline \multirow{2}{*}{ Tiene pareja } & Sí & 21 & 80,8 & 21 & 80,8 & \multirow{2}{*}{0,000} & \multirow{2}{*}{1,000} \\
\hline & No & 5 & 19,2 & 5 & 19,2 & & \\
\hline \multirow{4}{*}{$\begin{array}{l}\text { Cuáles son las personas } \\
\text { que la apoyan en el } \\
\text { cuidado de sus hijos }\end{array}$} & Ninguno & 12 & 46,2 & 2 & 7,7 & \multirow{4}{*}{11,198} & \multirow{4}{*}{$0,011 *$} \\
\hline & Pareja & 5 & 19,2 & 13 & 50,0 & & \\
\hline & Padres & 6 & 23,1 & 6 & 23,1 & & \\
\hline & Otros & 3 & 11,5 & 5 & 19,2 & & \\
\hline \multirow{2}{*}{$\begin{array}{l}\text { Ha considerado } \\
\text { aplazar o abandonar } \\
\text { su formación por la } \\
\text { maternidad }\end{array}$} & Sí & 20 & 76,9 & 15 & 57,7 & \multirow[b]{2}{*}{2,185} & \multirow[b]{2}{*}{0,139} \\
\hline & No & 6 & 23,1 & 11 & 42,3 & & \\
\hline \multirow{2}{*}{$\begin{array}{l}\text { Ha aplazado o } \\
\text { abandonado su } \\
\text { formación por la } \\
\text { maternidad }\end{array}$} & Sí & 18 & 69,2 & 13 & 50,0 & \multirow{2}{*}{1,997} & \multirow{2}{*}{0,158} \\
\hline & No & 8 & 30,8 & 13 & 50,0 & & \\
\hline \multirow{2}{*}{$\begin{array}{l}\text { Tiene apoyo familiar en } \\
\text { el cuidado de sus hijos }\end{array}$} & Sí & 17 & 65,4 & 23 & 88,5 & \multirow[t]{2}{*}{3,900} & \multirow[t]{2}{*}{$0,048 *$} \\
\hline & No & 9 & 34,6 & 3 & 11,5 & & \\
\hline
\end{tabular}

*Valor $\mathrm{P}=<0,05$

En la tabla 2 se describen los resultados de la variable de estrés académico de las participantes, en los cuales se puede observar que la diferencia más significativa entre el grupo de casos y el grupo de control se da en el estrés psicológico. La mediana que se presentan en el grupo de casos es de 9,50 y la mediana del grupo de control es de 12,50 con un coeficiente de significación de 0,037 , lo que permite observar un mayor nivel de estrés psicológico en el grupo de control. 
Tabla 2. Resultados del estrés académico

\begin{tabular}{ccccc}
\hline \multirow{2}{*}{ Estrés } & Grupo Casos & Grupo Control & $\begin{array}{c}\text { Prueba U de } \\
\text { Mann- Whitney }\end{array}$ & $\begin{array}{c}\text { Significación } \\
\text { asintótica }\end{array}$ \\
\cline { 2 - 3 } Mediana & Mediana & Mann & $0,037^{*}$ \\
Psicológico & $9,50(5,5)$ & $12,50(5,5)$ & 224,5 & 0,183 \\
Fomportamental & $7,00(5,0)$ & $8,50(4,5)$ & 265,5 & 0,108 \\
\hline
\end{tabular}

*Valor $\mathrm{P}=<0,05$

De acuerdo con los resultados obtenidos en la tabla 3, y teniendo en cuenta el coeficiente de significación estadística, se puede establecer que no hay diferencias significativas en los niveles de resiliencia de ambos grupos.

Tabla 3. Resultados de resiliencia

\begin{tabular}{|c|c|c|c|c|c|c|c|}
\hline \multirow{2}{*}{$\begin{array}{c}\text { Resiliencia } \\
\text { (Categorías) }\end{array}$} & \multirow{2}{*}{ Respuesta } & \multicolumn{2}{|c|}{ Grupo Casos } & \multicolumn{2}{|c|}{ Grupo Control } & \multirow{2}{*}{ Chi $\chi^{2}$} & \multirow{2}{*}{$\begin{array}{c}\text { Significación } \\
\text { asintótica }\end{array}$} \\
\hline & & $\mathbf{N}$ & $\%$ & $\mathbf{n}$ & $\%$ & & \\
\hline & Bajo & 1 & 3,8 & 1 & 3,80 & & \\
\hline \multirow[t]{3}{*}{ Resiliencia total } & Promedio & 12 & 46,2 & 13 & 50 & 0,08 & 0,961 \\
\hline & Alto & 13 & 50,0 & 12 & 46,2 & & \\
\hline & Bajo & 0 & 0,0 & 2 & 7,7 & & \\
\hline \multirow[t]{3}{*}{ Identidad } & Promedio & 5 & 19,2 & 6 & 23,1 & 2,322 & 0,313 \\
\hline & Alto & 21 & 80,8 & 18 & 69,2 & & \\
\hline & Bajo & 1 & 3,8 & 4 & 15,4 & & \\
\hline \multirow[t]{3}{*}{ Autonomía } & Promedio & 6 & 23,1 & 8 & 30,8 & 2,843 & 0,241 \\
\hline & Alto & 19 & 73,1 & 14 & 53,8 & & \\
\hline & Bajo & 1 & 3,8 & 1 & 3,8 & & \\
\hline \multirow[t]{3}{*}{ Satisfacción } & Promedio & 8 & 30,8 & 10 & 38,5 & 0,347 & 0,841 \\
\hline & Alto & 17 & 65,4 & 15 & 57,7 & & \\
\hline & Bajo & 2 & 7,7 & 0 & 0,0 & & \\
\hline \multirow[t]{3}{*}{ Pragmatismo } & Promedio & 8 & 30,8 & 10 & 38,5 & 2,222 & 0,329 \\
\hline & Alto & 16 & 61,5 & 16 & 61,5 & & \\
\hline & Bajo & 2 & 7,7 & 5 & 19,2 & & \\
\hline \multirow[t]{3}{*}{ Vínculos } & Promedio & 12 & 46,2 & 12 & 46,2 & 1,714 & 0,424 \\
\hline & Alto & 12 & 46,2 & 9 & 34,6 & & \\
\hline & Bajo & 1 & 3,8 & 2 & 7,7 & & \\
\hline \multirow[t]{2}{*}{ Redes } & Promedio & 7 & 26,9 & 6 & 23,1 & 0,410 & 0,815 \\
\hline & Alto & 18 & 69,2 & 18 & 69,2 & & \\
\hline
\end{tabular}




\begin{tabular}{|c|c|c|c|c|c|c|c|}
\hline \multirow{2}{*}{$\begin{array}{l}\text { Resiliencia } \\
\text { (Categorías) }\end{array}$} & \multirow{2}{*}{ Respuesta } & \multicolumn{2}{|c|}{ Grupo Casos } & \multicolumn{2}{|c|}{ Grupo Control } & \multirow{2}{*}{ Chi $\chi^{2}$} & \multirow{2}{*}{$\begin{array}{c}\text { Significación } \\
\text { asintótica }\end{array}$} \\
\hline & & $\mathbf{N}$ & $\%$ & $\mathbf{n}$ & $\%$ & & \\
\hline \multirow{3}{*}{ Modelos } & Bajo & 2 & 7,7 & 5 & 19,2 & & \\
\hline & Promedio & 11 & 42,3 & 12 & 46,2 & 2,056 & 0,358 \\
\hline & Alto & 13 & 50,0 & 9 & 34,6 & & \\
\hline \multirow{3}{*}{ Metas } & Bajo & 0 & 0,0 & 0 & 0,0 & & \\
\hline & Promedio & 14 & 53,8 & 12 & 46,2 & 0,308 & 0,579 \\
\hline & Alto & 12 & 46,2 & 14 & 53,8 & & \\
\hline \multirow{3}{*}{ Afectividad } & Bajo & 5 & 19,2 & 3 & 11,5 & & \\
\hline & Promedio & 10 & 38,5 & 11 & 42,3 & 0,591 & 0,744 \\
\hline & Alto & 11 & 42,3 & 12 & 46,2 & & \\
\hline \multirow{3}{*}{ Autoeficacia } & Bajo & 2 & 7,7 & 2 & 7,7 & & \\
\hline & Promedio & 14 & 53,8 & 11 & 42,3 & 0,751 & 0,687 \\
\hline & Alto & 10 & 38,5 & 13 & 50,0 & & \\
\hline \multirow{3}{*}{ Aprendizaje } & Bajo & 1 & 3,8 & 2 & 7,7 & & \\
\hline & Promedio & 17 & 65,4 & 10 & 38,5 & 3,785 & 0,151 \\
\hline & Alto & 8 & 30,8 & 14 & 53,8 & & \\
\hline \multirow{3}{*}{ Generatividad } & Bajo & 1 & 3,8 & 0 & 0,00 & & \\
\hline & Promedio & 8 & 30,8 & 8 & 30,8 & 1,029 & 0,598 \\
\hline & Alto & 17 & 65,4 & 18 & 69,2 & & \\
\hline
\end{tabular}

Por otra parte, la tabla 4 muestra las correlaciones entre el estrés académico y la resiliencia en ambos grupos, siendo significativa en el grupo control, lo cual indica que solamente para este grupo hay una relación entre las variables de estrés psicológico, comportamental y físico y el nivel total de resiliencia. La correlación entre estas dos variables es inversa; por lo tanto, a menores niveles de resiliencia mayor estrés académico.

Tabla 4. Asociación entre las variables de estrés académico y resiliencia.

\begin{tabular}{cccccc}
\hline \multirow{2}{*}{ Variable } & \multicolumn{2}{c}{ Grupo Casos } & \multicolumn{2}{c}{ Grupo Control } \\
\cline { 2 - 5 } & $\begin{array}{c}\text { Coeficiente de } \\
\text { correlación }\end{array}$ & $\begin{array}{c}\text { Sig. } \\
\text { (bilateral) }\end{array}$ & $\begin{array}{c}\text { Coeficiente de } \\
\text { correlación }\end{array}$ & $\begin{array}{c}\text { Sig. } \\
\text { (bilateral) }\end{array}$ \\
\hline Estrés psicológico & 0,044 & 0,831 &,$- 478^{*}$ & 0,013 \\
Estrés comportamental & $-0,266$ & 0,189 &,$- 741^{*}$ & 0,000 \\
Estrés físico & $-0,247$ & 0,225 &,$- 461^{*}$ & 0,018 \\
\hline
\end{tabular}

*Valor $\mathrm{P}=<0,05$ 


\section{Discusión y conclusiones}

El propósito de este estudio fue determinar la asociación entre los niveles de estrés académico y los niveles de resiliencia en madres universitarias con y sin apoyo por parte de la institución de educación superior en el cuidado de sus hijos. Los resultados mostraron que el grupo que no tenía apoyo institucional presenta mayores niveles de estrés psicológico que el grupo que sí recibe apoyo. En cuanto a los niveles de resiliencia, no se encontraron diferencias estadísticamente significativas en ambos grupos; sin embargo, se pudo observar que en el grupo de quienes no reciben apoyo institucional, la resiliencia tuvo una correlación inversa con el estrés académico, evidenciando que a menores niveles de resiliencia mayor estrés académico en todos los dominios que mide el instrumento: psicológico, comportamental y físico.

La explicación de lo anterior podría ser esta: el grupo de control, al no tener apoyo institucional, se considera más expuesto al estrés y, aunque de manera general, contrastando ambos grupos, los resultados de resiliencia no evidencian una diferencia estadísticamente significativa, sí se logra observar que en varios factores que componen la resiliencia hay una tendencia a una puntuación más baja en el grupo control.

En relación con lo anterior Villalta (2009), en su estudio sobre los factores de resiliencia asociados al rendimiento académico en estudiantes de contextos de alta vulnerabilidad social, utilizando también la Escala de Resiliencia (SV-RES), encontró una correlación entre los factores de resiliencia de identidad, metas y generatividad con el rendimiento académico en estudiantes que reportaban divorcio y separación de sus padres y las que se encontraban en embarazo o desempeñaban simultáneamente los roles de madre y estudiante.

En esta misma línea, García-León et al. (2019) encontraron que la resiliencia puede estar asociada con eventos estresantes de la vida, dado que los individuos se perciben menos resilientes cuando los eventos más estresantes les afectan, y las personas con niveles de resiliencia más bajos muestran altos niveles de síntomas psicopatológicos.

En cuanto al estrés, los hallazgos de esta investigación mostraron que en el grupo de control los niveles de estrés psicológico son superiores al grupo de casos, lo cual se refiere a las actitudes o reacciones frente a situaciones estresantes que se manifiestan en inquietud, incapacidad de relajarse o estar tranquilo, sentimientos de depresión y tristeza, ansiedad, angustia o desesperación, problemas de concentración, sentimiento de agresividad o aumento de irritabilidad. Castillo et al. (2016) encontraron altos niveles de estrés en estudiantes universitarios chilenos, especialmente frente a 
sobrecarga académica, falta de tiempo para cumplir con las actividades académicas y realización de un examen.

Conforme se incrementa la presencia femenina en las universidades, para estas se convierte en un reto la permanencia de las estudiantes madres. Por esto, no deja de llamar la atención en los resultados del presente estudio que el $76,9 \%$ de las madres del grupo de casos y el 57,7\% del grupo de control han considerado abandonar o aplazar sus estudios y el 69,2\% del grupo de casos y el $50 \%$ del grupo de control lo han hecho en algún momento de sus carreras. Al respecto Miller y Arvizu (2016) afirman que la maternidad condiciona la planeación de la vida en las mujeres estudiantes, pues deben atender simultáneamente responsabilidades con sus estudios, con sus familias e incluso con sus trabajos.

Acerca de este tema, las teorías sobre la resiliencia afirman que esta puede ser entendida como una adaptación exitosa en los individuos que se han visto sometidos a situaciones estresantes, lo cual se puede lograr disminuyendo la exposición a circunstancias de vida que atenten contra la salud mental e incrementando los factores protectores que pueden estar presentes en un ambiente determinado, por ejemplo generando o fortaleciendo fuentes de apoyo y estimulando la adquisición de diversas habilidades (Kotliarenco et al., 1997).

De esta manera, los resultados hallados en la presente investigación sugieren que el apoyo que se ofrece en la institución de educación superior del grupo casos tiene efectos positivos para sus beneficiarias, dado que el apoyo social les posibilita potencializar su resiliencia. Puesto que la maternidad en el ámbito universitario es un fenómeno creciente, es preciso que siga siendo estudiado para que se establezcan los factores relevantes que garanticen una experiencia y unas condiciones positivas en las mujeres que se desempeñan en este ambiente, para así permitir la identificación de los factores que contribuyen en la deserción universitaria, aplazamiento de la formación profesional o bajo rendimiento académico y para contribuir en el avance en la equidad institucional, un desafío fundamental en las instituciones de educación superior.

Una limitante del presente estudio es el tamaño reducido de la muestra, que puede producir algunos sesgos en los resultados, por lo cual se considera que podría verse enriquecido en lo porvenir con la participación de otras instituciones de educación superior de carácter público y privado para apreciar otras variables que afectan el estrés académico en las mujeres madres universitarias. 


\section{Agradecimientos}

Las autoras les agradecen al Instituto Tecnológico Metropolitano y a la Institución Universitaria de Envigado por posibilitar el contacto con las participantes y a ellas, las madres estudiantes.

\section{Referencias}

Álamo, N. A., Krause, M., Pérez, J. C. y Aracena, M. (2017). Impacto de la Salud Psicosocial de la madre adolescente en la relación con el niño/a y su desarrollo. Revista argentina de Clínica Psicológica, 26(3), 332-346. https://doi. org/10.24205/03276716.2017.1022

Barraza, A. (2007). Propiedades psicométricas del Inventario SISCO del estrés académico. Revista PsicologíaCientífica.com, 9(10). http://www. psicologiacientifica.com/sisco-propiedades-psicometricas

Barraza, A. y Silerio, J. (2007). El estrés académico en alumnos de educación media superior: un estudio comparativo. Revista Investigación Educativa, 7, 48-65. https://www.researchgate.net/publication/28175059_El_estres_academico_en_ los_alumnos_de_educacion_media_superior_Un_estudio_comparativo

Berrío, N. y Mazo, R. (2011). Estrés Académico. Revista de Psicología Universidad de Antioquía, 3(2), 65-82. https://revistas.udea.edu.co/index.php/psicologia/ article/view/11369

Castillo, C., Chacón, T. y Díaz-Véliz, G. (2016). Ansiedad y fuentes de estrés académico en estudiantes de carreras de la salud. Investigación en Educación Médica, 5, 230 237. https://doi.org/10.1016/J.RIEM.2016.03.001

CEPAL. (1998). La educación de las mujeres: De la marginalidad a la coeducación: propuestas para una metodología de cambio educativo = The education of women: from marginality to coeducation: proposals for a methodology to achieve educational change. CEPAL.

Congreso de la República de Colombia. (2006, 8 de noviembre). Ley 1098 de 2006. Por la cual se expide el Código de la Infancia y la Adolescencia. Diario Oficial N. ${ }^{\circ}$ 46.446.

Congreso de la República de Colombia. (2006, 6 de septiembre). Ley 1090 de 2006. Por la cual se reglamenta el ejercicio de la profesión de Psicología, se dicta el Código Deontológico y Bioético y otras disposiciones". Diario Oficial N. ${ }^{\circ} 46.383$.

Departamento Administrativo Nacional de Estadística y onu Mujeres. (2020). Tiempo de cuidados: Las cifras de la desigualdad. Las mujeres cuentan. https://www.dane. 
gov.co/files/investigaciones/genero/publicaciones/tiempo-de-cuidados-cifrasdesigualdad-informe.pdf

Domínguez, G., Martínez, A., Herrera, H. y Rincón, M. E. (2018). Impacto personal y escolar del embarazo y maternidad en estudiantes universitarios. Investigación y Práctica en Psicología Del Desarrollo, 1, 167-174. https://doi.org/10.33064/ ippd1644

Estupiñán, M. R. y Vela, D. R. (2012). Calidad de vida de madres adolescentes estudiantes universitarias. Revista Colombiana de Psiquiatría, 41(3), 536-549. https://doi.org/10.1016/S0034-7450(14)60027-5

Feder, A., Nestler, E. J. y Charney, D. S. (2009). Psychobiology and molecular genetics of resilience. Nature reviews: Neuroscience, 10(6), 446-457. https://doi. org/10.1038/nrn2649

García-León, M. Á., Pérez-Mármol, J. M., González-Pérez, R., García-Ríos, M. y Peralta-Ramírez, M. I. (2019). Relationship between resilience and stress: Perceived stress, stressful life events, HPA axis response during a stressful task and hair cortisol. Physiology \& behavior, 202, 87-93. https://doi.org/10.1016/j. physbeh.2019.02.001

González-Olaya, H. L., Delgado-Rico, H. D., Escobar-Sánchez, M. y CárdenasAngelone, M. E. (2014). Asociación entre el estrés, el riesgo de depresión y el rendimiento académico en estudiantes de los primeros semestres de un programa colombiano de medicina. FEM: Revista de la Fundación Educación Médica, 17(1), 47-54. https://doi.org/10.4321/S2014-98322014000100008

Instituto Tecnológico Metropolitano. (s.f.). Aula Pedagógica Infantil. Consultado el 30 de mayo de 2019. https://www.itm.edu.co/dependencias/bienestarinstitucional-4/proyecto-aula-pedagogica-infantil/

Kotliarenco, M. A., Cáceres, I. y Fontecilla, M. (1997). Estado de Arte en Resiliencia. Organización Panamericana de la Salud.

Lazarus, R. S. y Folkman, S. (1986). Estrés y procesos cognitivos. Martínez Roca.

Malo, D. A., Cáceres, G. S. y Peña, G. H. (2010). Validación del inventario SISCO del estrés académico y análisis comparativo en adultos jóvenes de la Universidad Industrial de Santander y la Universidad Pontificia Bolivariana, Seccional Bucaramanga, Colombia. Praxis Investigativa ReDIE: revista electrónica de la Red Durango de Investigadores Educativos, 2(3), 26-42.

Martínez, E. S. y Díaz, D. A. (2007). Una aproximación psicosocial al estrés escolar. Educación y educadores, 10(2), 11-22. 
Maturana, H. A. y Vargas, S. A. (2015). El estrés escolar. Revista Médica Clínica Las Condes, 26(1), 34-41. https://doi.org/10.1016/j.rmclc.2015.02.003

Miller, D. y Arvizu, V. (2016). Ser madre y estudiante. Una exploración de las características de las universitarias con hijos y breves notas para su estudio. Revista de la Educación Superior, 45(177), 17-42. https://doi.org/10.1016/j. resu.2016.04.003

Ministerio de Salud. (1993, 4 de octubre). Resolución 8430 de 1993. Por la cual se establecen las normas científicas, técnicas y administrativas para la investigación en salud.

Oken, B. S., Chamine, I. y Wakeland, W. (2015). A Systems Approach to Stress, Stressors and Resilience in Humans. Behavioural brain research, 0, 144-154. https://doi.org/10.1016/j.bbr.2014.12.047

Restrepo, J. E., Sánchez, O. A. y Castañeda-Quirama, T. (2020). Estrés académico en estudiantes universitarios. Psicoespacios, 14(24), 17-37. http://revistas.iue.edu. co/revistasiue/index.php/Psicoespacios/article/view/1331

Rodríguez, J., Millanes, B. y Durand, J. (2019). Universidad y maternidad: Madres universitarias en la Universidad de Sonora. Universidades, 70(79), 41-52. https:// doi.org/10.36888/udual.universidades.2019.79.64

Russo, S. J., Murrough, J. W., Han, M. H., Charney, D. S. y Nestler, E. J. (2012). Neurobiology of resilience. Nature neuroscience, 15(11), 1475-1484. https://doi. org/10.1038/nn.3234

Saavedra, E. y Villalta, M. (2008). Escala de resiliencia SV-RES para jóvenes y adultos. Ceanim.

Sapolsky, R. (2008). ¿Por qué las cebras no tienen úlcera?: La guía del estrés. Alianza Editorial.

Steen, M. y Francisco, A. A. (2019). Salud mental y bienestar materno. Acta Paulista de Enfermagem, 32(4), III-IV. https://doi.org/10.1590/1982-0194201900049

Urbano, F. A., Oiene, S. E., Cruz, P. D., Aragón, D. E., Guantay, L. F., Salom, M. G. y Torres, D. B. (2012). Resiliencia, maternidad y expectativas de vida en alumnas universitarias. Cuadernos Universitarios, 5, 141-147. http://revistas.ucasal.edu. ar/index.php/CU/article/view/101

Villalta, M. (2010). Factores de resiliencia asociados al rendimiento académico en estudiantes de contextos de alta vulnerabilidad social. Revista de pedagogía, 31(88), 159-188. https://www.redalyc.org/articulo.oa?id=65916617007 